\title{
New Approaches to the Diagnosis and Treatment of Cryptococcal Meningitis
}

\author{
Azure T. Makadzange, MD, DPhil ${ }^{1,2}$ Grace McHugh, $\mathrm{MBBS}^{3}$
}

\footnotetext{
${ }^{1}$ Department of Ragon Institute of MGH, MIT and Harvard, Medicine Harvard Medical School, Boston, Massachusetts

2 Department of Medicine, University of Zimbabwe College of Health Sciences, Harare, Zimbabwe

${ }^{3}$ Department of Medicine, Parirenyatwa Hospital Family Care Centre, University of Zimbabwe College of Health Sciences,

Harare, Zimbabwe
}

Address for correspondence Azure T. Makadzange, MD, DPhil, Department of Ragon Institute of MGH, MIT and Harvard, Medicine Harvard Medical School, 400 Technology Square, Boston, MA 02139 (e-mail: amakadzange@mgh.harvard.edu).

Semin Neurol 2014;34:47-60.

\author{
Abstract \\ Keywords \\ - cryptococcal \\ meningitis \\ - human \\ immunodeficiency \\ virus \\ - acquired \\ immunodeficiency \\ syndrome \\ - sub-Saharan Africa
}

Cryptococcal meningitis remains one of the leading causes of morbidity and mortality among immunosuppressed individuals, particularly those with advanced acquired immunodeficiency syndrome. The greatest burden of disease is in sub-Saharan Africa and Asia where there is limited access to diagnostics and treatment for the disease. The authors review the available tools for diagnosing cryptococcal meningitis and review treatment for cryptococcal meningitis, highlighting the evidence behind current treatment guidelines.
The global burden of cryptococcal disease is high with 1 million new infections each year. ${ }^{1}$ The greatest burden is in subSaharan Africa where cryptococcal meningitis is the fourth leading cause of mortality, accounting for over 600,000 deaths annually. ${ }^{1}$ Disseminated cryptococcosis is the leading cause of meningitis in Zimbabwe, ${ }^{2}$ and accounts for $40 \%$ of all cases of meningitis in Malawi. ${ }^{3}$ Cryptococcal meningitis is also associated with very high mortality rates, particularly in resource limited settings. ${ }^{4,5}$ Cryptococcosis is caused by an encapsulated yeast that belongs to the genus Cryptococcus. Cryptococcus neoformans and Cryptococcus gattii are responsible for the majority of cases of human cryptococcosis. Cryptococcus neoformans was originally classified into five groups (A-D and A/ D) based on serology. Serologic typing classified C. gattii as $C$. neoformans serotype $B$ and $C$, whereas serotypes $A$ and $D$ were C. neoformans var. grubii and $C$. neoformans var. neoformans, respectively. Molecular typing has enabled us to distinguish $C$. neoformans and $C$ gattii as two distinct species. Each species has been further divided into four major molecular subtypes, often with distinct ecological niches (- Table 1). Cryptococcus neoformans has a global distribution and has been found in association with pigeon droppings, soil, and decaying vegetation. ${ }^{6-8}$ Cryptococcus gattii has primarily been isolated in tropical and subtropical climates and is found in association with eucalyptus trees. ${ }^{9-12}$ Other Cryptococcus species that rarely cause human disease include $C$. albudius, ${ }^{13,14}$ C. laurentii, ${ }^{15,16}$ and C. luteolus. ${ }^{17}$

\section{Epidemiology of Cryptococcal Disease}

Cryptococcus was identified as a cause of human disease in 1894 when the organism was isolated from the tibia of a 31-year-old woman with disseminated disease. The first described case of meningoencephalitis was in 1905 by von Hansemann. Prior to the advent of the acquired immunodeficiency syndrome (AIDS) epidemic, cryptococcus rarely caused significant human disease. The onset of the AIDS epidemic resulted in a substantial increase in the number
Issue Theme HIV Neurology; Guest Editors, Serena Spudich, MD, MA, and Ana-Claire Meyer, MD, MSHS
DOI http://dx.doi.org/ 10.1055/s-0034-1372342. ISSN $0271-8235$. 
Table 1 Geographic distribution of cryptococcal species

\begin{tabular}{|l|l|l|l|l|l|}
\hline Species & Serotype & $\begin{array}{l}\text { Molecular } \\
\text { type }\end{array}$ & $\begin{array}{l}\text { Global } \\
\text { distribution }\end{array}$ & Disease & Source \\
\hline $\begin{array}{l}\text { C. Neoformans } \\
\text { var grubii }\end{array}$ & A & $\begin{array}{l}\text { VNI } \\
\text { VNII }\end{array}$ & Worldwide & $\begin{array}{l}\text { Immunocompromised } \\
\text { Pulmonary/ } \\
\text { meningitis/ } \\
\text { disseminated }\end{array}$ & Soil/avian environments \\
\hline C. gattii & B & $\begin{array}{l}\text { VGI } \\
\text { VGII } \\
\text { VGIII } \\
\text { VGIV }\end{array}$ & $\begin{array}{l}\text { Tropical, } \\
\text { subtropical, } \\
\text { temperate }\end{array}$ & $\begin{array}{l}\text { Immunocompromised/ } \\
\text { Immunocompetent } \\
\text { Less commonly } \\
\text { causes meningitis }\end{array}$ & Woody materials, i.e., trees \\
\hline $\begin{array}{l}\text { C. Neoformans } \\
\text { var neoformans }\end{array}$ & D & VNIV & Europe & $\begin{array}{l}\text { Immunocompromised/ } \\
\text { immunocompetent } \\
\text { Skin disease }\end{array}$ & Soil/avian environments \\
\hline C. Neoformans & A/D & VNIII & Worldwide & $\begin{array}{l}\text { Immunocompromised } \\
\text { Pulmonary/meningitis/ } \\
\text { disseminated }\end{array}$ & \\
\hline
\end{tabular}

of cases of cryptococcal disease. In the United States in the pre-HAART (highly active antiretroviral treatment) era, $86 \%$ of cryptococcosis cases occurred in human immunodeficiency virus- (HIV-) infected individuals, and the annual incidence of the disease was markedly higher in individuals with HIV (up to 66 cases/1,000) compared with those without HIV (0.9 cases/100,000). ${ }^{18}$ Among individuals who did not have $\mathrm{HIV}$, the greatest risk factors for developing cryptococcosis were malignancies, diabetes, steroid therapy, solid-organ transplant, and chronic medical diseases such as renal and liver failure. ${ }^{18}$ The introduction of HAART subsequently led to a substantial decline in incident cryptococcal infections in the developed world, ${ }^{19-21}$ though the prevalence among other immunocompromised individuals has remained stable. ${ }^{20}$

In sub-Saharan Africa, cryptococcal disease continues to be one of the leading causes of mortality among individuals with HIV infection. Among adults with HIV in South Africa, the estimated incidence is 95 to 120 cases per $100,000^{22,23}$; among individuals with AIDS (defined by CD4 $<200$ cells/ $\mathrm{mm}^{3}$ ) the incidence is 14 cases per $1,000{ }^{22}$ In southeast Asia there are over 100,000 annual cases of cryptococcal disease. ${ }^{1}$ The greatest burden of cryptococcal disease in Asia occurs in patients with AIDS, with cryptococcal meningoencephalitis accounting for up to a third of patients presenting with meningitis. ${ }^{24}$ The epidemic of cryptococcal disease in subSaharan Africa is slowly evolving with some areas experiencing a decline in the number of cases, while other areas continue to see a large number of cases despite increasing access to ART. ${ }^{25,26}$

\section{Host Susceptibility to Cryptococcal Disease}

Cryptococcus yeasts are ubiquitous in the environment and are acquired by inhalation. Primary pulmonary infection is likely acquired in childhood in a large proportion of individuals, ${ }^{27-29}$ although adult acquisition is also well documented. ${ }^{30,31}$ An effective cell-mediated immune response is important for recruiting and activating macrophages to con- tain disease, ${ }^{32,33}$ resulting in clearance or establishment of a contained latent infection. A Th1 predominant cellular immune response with production of interferon- (IFN-) $\gamma$, tumor necrosis factor- (TNF-) $\alpha$, interleukin- (IL-)12, and IL-18 is important for decreasing fungal burden and preventing dissemination of disease. ${ }^{34-36}$ A Th2 response, however, is associated with disseminated disease. ${ }^{37}$

In the immunocompromised host, reactivation of latent disease is believed to be responsible for the subsequent development of cryptococcal disease. ${ }^{38,39}$ In patients with AIDS, the depletion of CD4+ T cells affects the ability of the immune response to adequately contain cryptococcal infection. HIV infection results in a shift from a Th1 cytokine phenotype to a predominant $\mathrm{Th} 2$ phenotype, which is associated with disseminated cryptococcal disease. ${ }^{37}$ In addition, HIV may infect alveolar macrophages, further limiting their ability to adequately contain cryptococcal infection. $^{40}$

\section{Clinical Presentation of Cryptococcal Disease}

The clinical presentation of cryptococcal disease is highly variable and can result in asymptomatic disease, localized pulmonary disease or disseminated disease. Disseminated disease can occur in any organ; however, there is a predilection for infection of the central nervous system (CNS), resulting in meningoencephalitis and occasionally causing focal intracerebral granulomas known as cryptococcomas. Patients with meningoencephalitis typically present with a severe headache. The headache may be present for several weeks to months, and can be accompanied by mental status changes, personality changes, fever, lethargy, and coma. Other complications of CNS involvement include hydrocephalus (both communicating and noncommunicating), papilledema that may lead to blindness, sudden onset of sensorineural deafness, cranial nerve palsies, motor and sensory deficits, cerebellar dysfunction, and seizures. - Table 2 summarizes some common clinical presentations of meningoencephalitis in 
Table 2 Clinical presentation of meningoencephalitis among HIV-infected hospitalized patients

\begin{tabular}{|l|l|l|l|}
\hline Signs and symptoms & $\begin{array}{l}\text { HIV positive, USA } \\
(\boldsymbol{n}=89), \boldsymbol{N}(\%)^{41}\end{array}$ & $\begin{array}{l}\text { HIV positive, } \\
\text { South Africa } \\
(\boldsymbol{n}=44) \boldsymbol{N}(\%)^{42}\end{array}$ & $\begin{array}{l}\text { HIV positive, } \\
\text { Zimbabwe } \\
\mathbf{N}(\%)^{43}\end{array}$ \\
\hline Headache & $65(73)$ & $37(84)$ & $73 / 76(96)$ \\
\hline Fever & $58(65)$ & $21(48)$ & $38 / 74(51)$ \\
\hline Cough or dyspnea & $28(31)$ & ND & $16 / 67(24)$ \\
\hline Nausea or vomiting & $37(42)$ & ND & $31 / 76(41)$ \\
\hline Focal neurologic deficits & $5(6)$ & $22(50)^{\mathrm{a}}$ & $6 / 80(8)$ \\
\hline Seizures & $4(4)$ & $7(16)$ & $8 / 75(11)$ \\
\hline Altered mentation & $25(28)$ & $16(36)$ & $10 / 67(15)^{\mathrm{b}}$ \\
\hline CSF parameters & \multicolumn{3}{l|}{} \\
\hline Opening pressure $\left(\geq 200 \mathrm{~mm} \mathrm{H}_{2} 0\right)$ & $33 / 50(66)$ & $\mathrm{ND}$ & $\mathrm{ND}$ \\
\hline Low glucose & $21 / 89(24)$ & $27 / 44(61)^{\mathrm{c}}$ & $\mathrm{ND}$ \\
\hline Protein $>45 \mathrm{mg} / \mathrm{dL}$ & $49 / 89(55)$ & $33 / 41(81)$ & ND \\
\hline WBC $\geq 20$ & $19 / 89(21)^{\mathrm{d}}$ & $22 / 41(54)^{\mathrm{e}}$ & ND \\
\hline India Ink positive & $64 / 87(74)$ & $40 / 43(93)$ & $76 / 89(85)$ \\
\hline
\end{tabular}

Abbreviations: CSF, cerebrospinal fluid; HIV, human immunodeficiency virus; ND, not determined; WBC, white blood count.

${ }^{a}$ Focal neurologic signs included cranial nerve palsies, papilledema, hemiplegia, blindness, deafness, paraplegia.

befined by a Glasgow Coma Score $<13$.

'Glucose $<2.2 \mathrm{mmol} / \mathrm{L}(40 \mathrm{mg} / \mathrm{dL})$.

${ }^{\mathrm{d}} \mathrm{WBC} \geq 20$ cells $/ \mathrm{mm}^{3}$.

eWBC $\geq 5$ cells $/ \mathrm{mm}^{3}$.

${ }^{f}$ Median values for CSF parameters were provided as median WBC count $5 / \mathrm{mm}^{3}$ (range $0-1250$, predominantly mononuclear cells), median glucose level $2.1 \mathrm{mmol} / \mathrm{L}$ (0.1-5.1), median protein level $1.1 \mathrm{~g} / \mathrm{dL}$ (range $0.1-10.6$ ).

three patient cohorts, in the United States, ${ }^{41}$ South Africa, ${ }^{42}$ and Zimbabwe. ${ }^{43}$

Clinical presentation in sub-Saharan Africa is often delayed, with many patients presenting with advanced disease characterized by focal neurologic deficits and seizures. Cryptococcal meningitis is often the first AIDS-defining illness in many patients who present for care. Headache is the most common symptom on presentation, with meningismus present in most patients. ${ }^{2,43}$ Elevations in intracranial pressure are common, and may be due to fungal obstruction of the cerebrospinal fluid (CSF) drainage channels by accumulation in the arachnoid villi and subarachnoid spaces, resulting in communicating hydrocephalus. ${ }^{44}$ High opening pressures are associated with poor clinical outcomes. ${ }^{45}$ Ocular involvement may also be present, and most commonly occurs as a result of high intracranial pressures. Patients present with oculomotor palsies, papilledema, and complete vision loss. ${ }^{46}$ Intraocular cryptococcosis is less frequent, and can cause endophthalmitis or direct invasion of the optic nerve. ${ }^{47}$

\section{Diagnosis of Cryptococcal Meningitis}

The diagnosis of cryptococcal disease can be made by direct visualization, histopathology, culture, and or detection of cryptococcal antigens in blood, CSF, and urine. Cryptococcus exists in the blood, CSF, and tissues as a single-celled organism with a characteristic polysaccharide capsule. Microscopic identification of the organism can be done using India ink staining or observation with a phase-contrast microscope. India ink staining has been the traditional method for identifying Cryptococcus organisms, particularly in resource limited settings. India ink stains the surrounding material blue, but not the fungal capsule, giving a characteristic "starry night" appearance. The sensitivity and specificity of India ink staining can be highly variable and often operator dependent, as lysed leukocytes can be mistaken for fungal elements. ${ }^{48,49}$ The organism can readily be cultured from most sites, using routine and automated culture systems.

Histopathologic diagnosis of Cryptococcus can be done with several readily available staining techniques. The Giemsa stain only partially stains the organisms and is not typically utilized. Hematoxylin and eosin does not stain the capsule and the yeast is only weakly stained. Gomori methamine silver stain binds to fungal aldehydes and stains the yeast a characteristic black color, without staining the capsule. Staining of the capsule can be done with mucicarmine or Alcian blue stains. The capsule takes up the red color of mucicarmine or the blue color of Alcian to facilitate visualization. In tissues, yeast are typically contained within granulomas, particularly in immunocompetent hosts. In immunocompetent individuals, the Cryptococci are taken up by macrophages and induce an inflammatory response with the formation of epitheloid granulomas with central giant cells and surrounding lymphocytes. These granulomas typically do not have caseous necrosis. ${ }^{50}$ In immunocompromised individuals, full granuloma formation may not occur, and pseudocysts develop that 
are filled with encapsulated yeasts and surrounded by macrophages and lymphocytes. ${ }^{44,50}$

An evaluation of CSF parameters, such as cell counts, glucose, protein, and opening pressures, can aid in the diagnostic evaluation of cryptococcal meningoencephalopathy. In a case series of 40 patients with cryptococcal meningitis in the setting of diabetes, malignancies, sarcoidosis, and other rheumatologic disease, most patients presented with abnormal CSF parameters. ${ }^{51}$ Abnormal CSF cell counts were present in 97\%. Cell counts ranged from 6 to 808 cells $/ \mathrm{mm}^{3}$, with lymphocytes accounting for 8 to $100 \%$ of CSF white cells. Protein elevation was present in $90 \%$, and a low CSF glucose in $55 \%$. The opening pressure was elevated in $64 \% .{ }^{51}$ In patients presenting with cryptococcal meningoencephalitis in the setting of advanced HIV infection, the classic findings of an elevated CSF white cell count, elevated protein, and low glucose are not always evident (-Table $\mathbf{2}$ ).

Detection of capsular antigen is the most reliable diagnostic tool for cryptococcosis. Cryptococcal antigen can be detected in serum, CSF, and urine specimens. Detection of capsular antigen can be done by latex agglutination (LA) assays, enzyme immunoassays (EIAs), or the novel lateral flow assay (LFA). The latex agglutination assay has been used for several decades for the detection of cryptococcal antigen and has a higher sensitivity and specificity than India ink staining. ${ }^{52,53}$ Latex particles are coated with anticryptococcal antibodies, and in the presence of cryptococcal antigen will agglutinate, forming visible clumps that are subjectively read on a predefined scale. The sensitivity and specificity of the LA tests vary with manufacturer and the use of pronase. ${ }^{54,55} \mathrm{~A}$ study comparing four commercially available LA tests and an EIA test to culture found a serum sensitivity of LA that ranged from 83 to $97 \%{ }^{54}$ The tests with the lowest sensitivity did not use pronase on serum specimens. The specificity of the LA on serum ranged from 93 to $100 \%$. The CSF sensitivity and specificity of the LA test was high and ranged from 93 to $100 \%$, while the specificity range was 93 to $98 \%$. The sensitivity and specificity on EIA testing of CSF was $100 \%$ and $98 \%$, respectively. ${ }^{54,56}$ The sensitivity and specificity of the EIA on serum was 93\% and 96\%, respectively. Although the LA performs well when compared with EIA and culture, ${ }^{54,56}$ its primary limitation is that it is a cumbersome manual test with subjectivity in the interpretation of the result. The test also requires laboratory equipment and refrigeration of reagents, making it unsuitable for use in remote resource limited settings.

In 2009, a rapid point of care diagnostic test known as the lateral flow assay (LFA) was developed. The Cryptococcal antigen ( $\mathrm{CrAg}$ ) LFA is a capillary flow sandwich immunochromographic assay that can be done as a point of care test. Goldconjugated anticryptococcal monoclonal antibodies and control goat IgG antibodies are deposited on a membrane on the test strip. Specimen diluent is added to a tube to which $40 \mu \mathrm{L}$ of the patient specimen is added. The test strip is added to the tube and incubated at room temperature for 10 minutes before the result is read. The specimen migrates by capillary flow up the test strip, and in the presence of cryptococcal antigen will bind to the anticryptococcal monoclonal anti- bodies. The bound antibodies will continue to flow up the dipstick to the detection lines. The first detection line contains immobilized anticryptococcal monoclonal antibodies, and the distal line contains immobilized bovine antigoat IgG antibodies. A gold-conjugated anticryptococcal antibody that is bound to cryptococcal antigen will bind to the first detection line containing anticryptococcal antibody, creating a sandwich that is detected as a visible line at the test line site. The control goat IgG antibodies will continue to migrate up the test strip and bind to the bovine antigoat IgG antibodies and be detected as a visible line in the control test line. A positive test is indicated by the presence of two lines (the test line and the control line); a negative test is indicated by the presence of the control line only. An invalid test is indicated by the absence of a control line. The sensitivity and specificity of the lateral flow assay is very high, with high levels of concordance with EIA and latex agglutination assays. ${ }^{57-59}$ In a study using archived specimens in Thailand, the LFA was positive in all blood culture positive specimens and had strong concordance with the EIA on testing of serum. ${ }^{58}$ The LFA has also been shown to have high sensitivity in detection of all serotypes (A-D). ${ }^{60,61}$ The test has been approved by the Food and Drug Administration for use on serum and CSF.

The introduction of the lateral flow assay has the potential to revolutionize cryptococcal diagnosis in resource limited settings. ${ }^{62}$ It is as simple to perform as a urine pregnancy test, and therefore does not require trained laboratory personnel. It can be performed in the field and does not require sophisticated laboratory equipment, refrigeration and/or centrifugation.

\section{Treatment of Cryptococcal Meningitis}

In the absence of therapy cryptococcal meningoencephalitis is uniformly fatal. ${ }^{4}$ Early diagnosis and prompt treatment is critical to improve survival. The classes of antifungal drugs that have activity against Cryptococcus are the polyenes (amphotericin B formulations), the azoles, and flucytosine. Treatment of cryptococcal meningitis typically consists of a 2-week induction phase of therapy followed by 8 weeks of consolidation therapy, and additional maintenance therapy that acts as secondary prophylaxis against recurrence. The recommended treatment regimens are indicated in - Table 3 .

Combination therapy with amphotericin B and flucytosine was established as the superior regimen in the mid1990s, and globally is the preferred regimen. ${ }^{63-65}$ However, both amphotericin B and flucytosine are associated with significant toxicities that included renal toxicity, anemia, and neutropenia, and require intravenous administration and monitoring of toxicities. In much of sub-Saharan Africa and parts of Asia flucytosine is either not registered or is too expensive for clinical use. Amphotericin B is not often available, particularly in remote settings far from central hospitals. The cost of amphotericin $\mathrm{B}$, the monitoring and management of its associated toxicities, and the prolonged hospital admission make it an expensive treatment option 


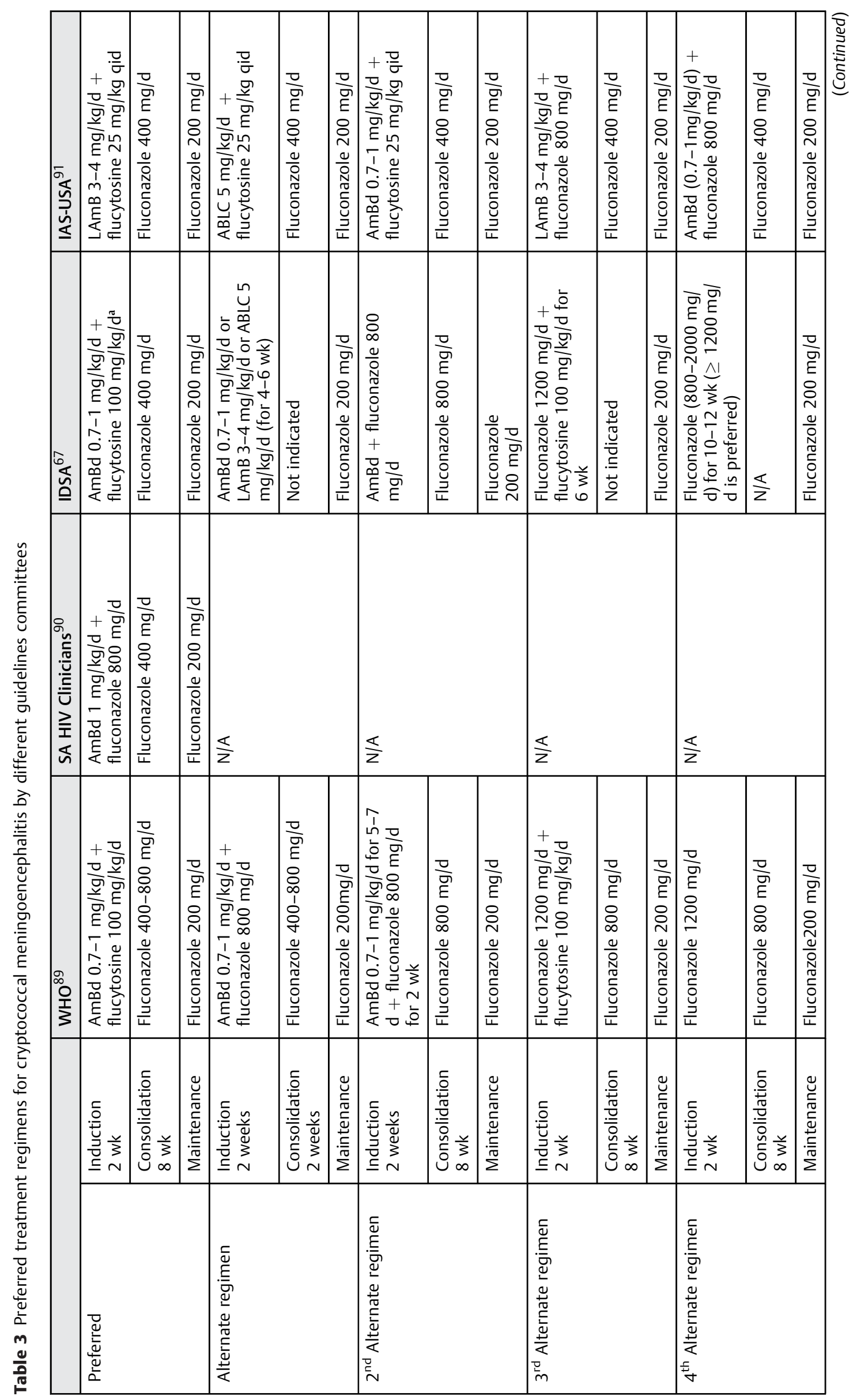




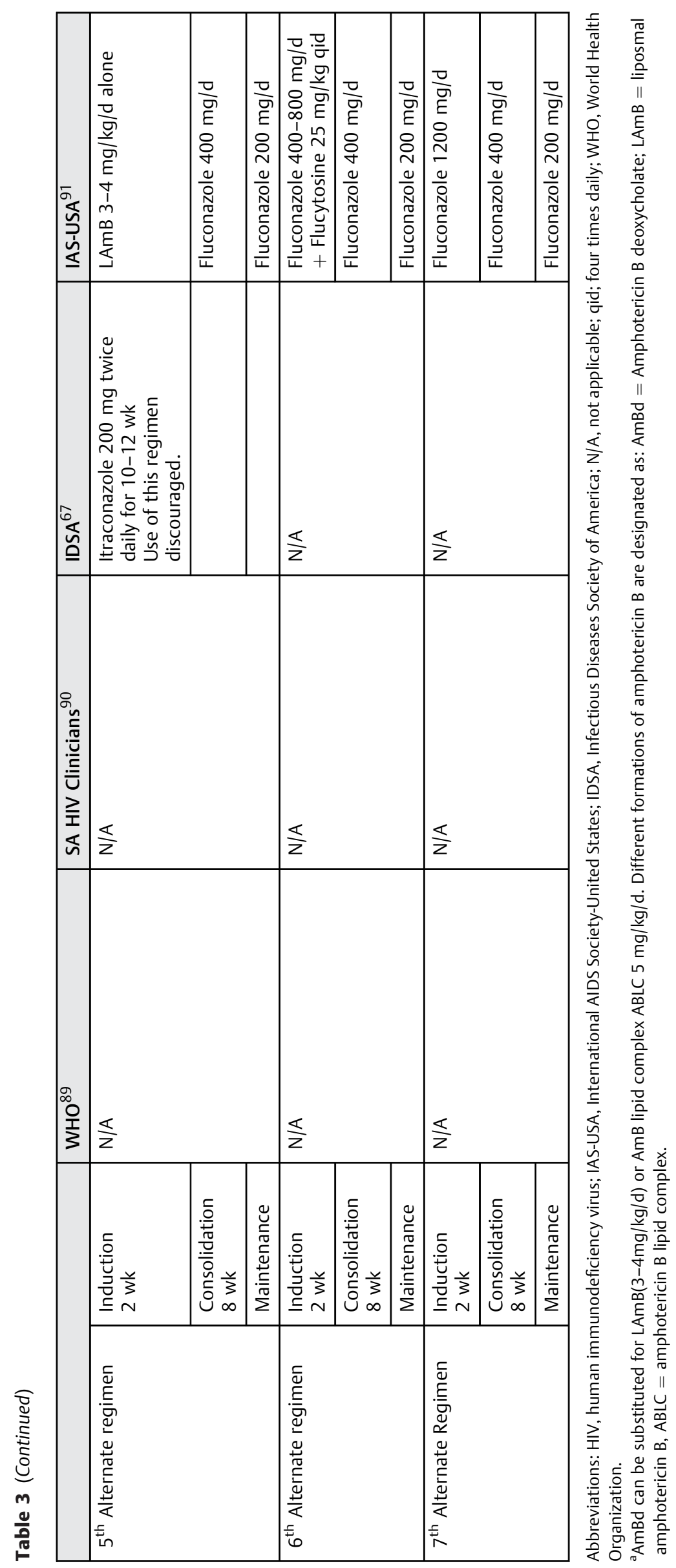




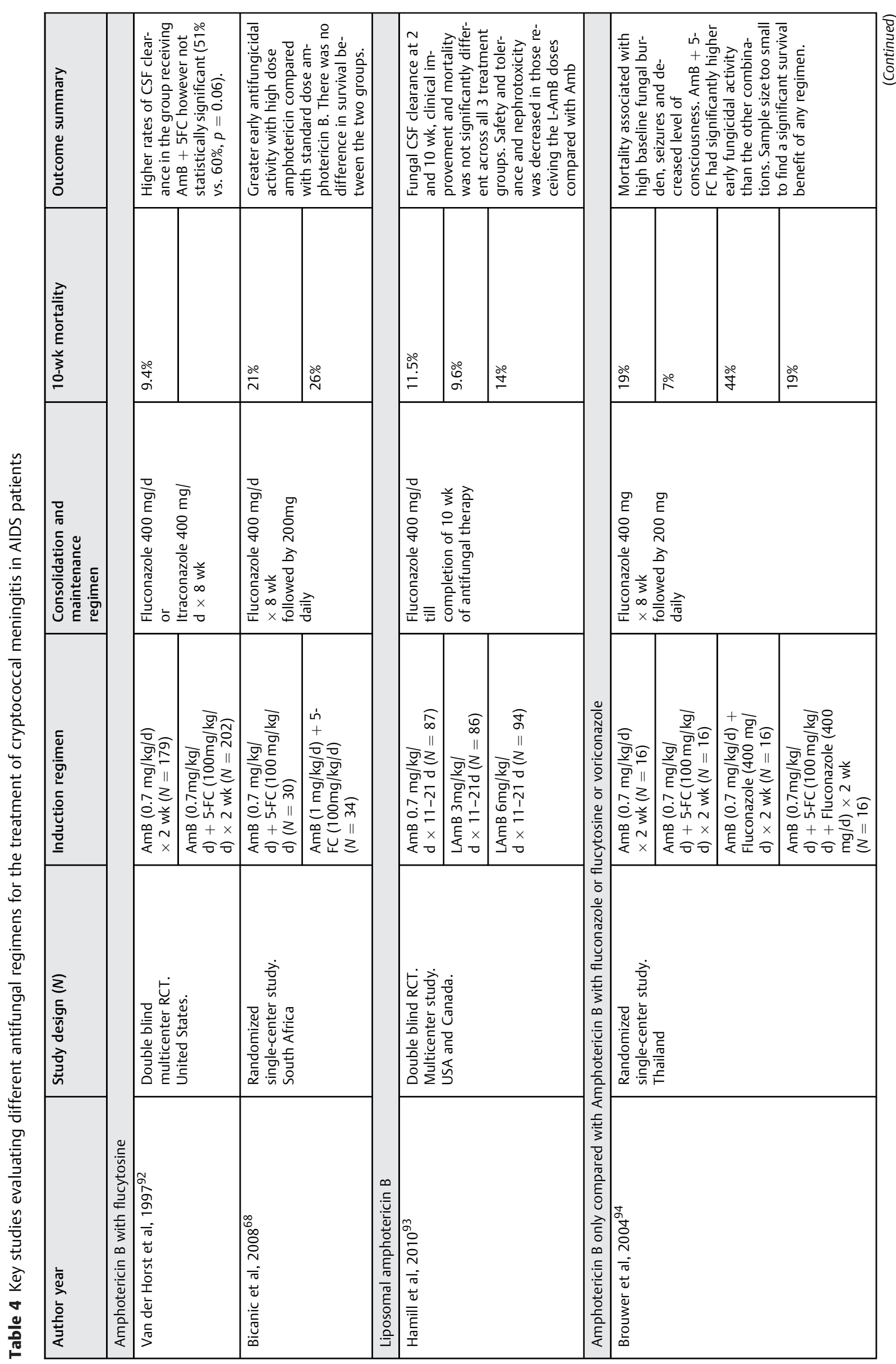




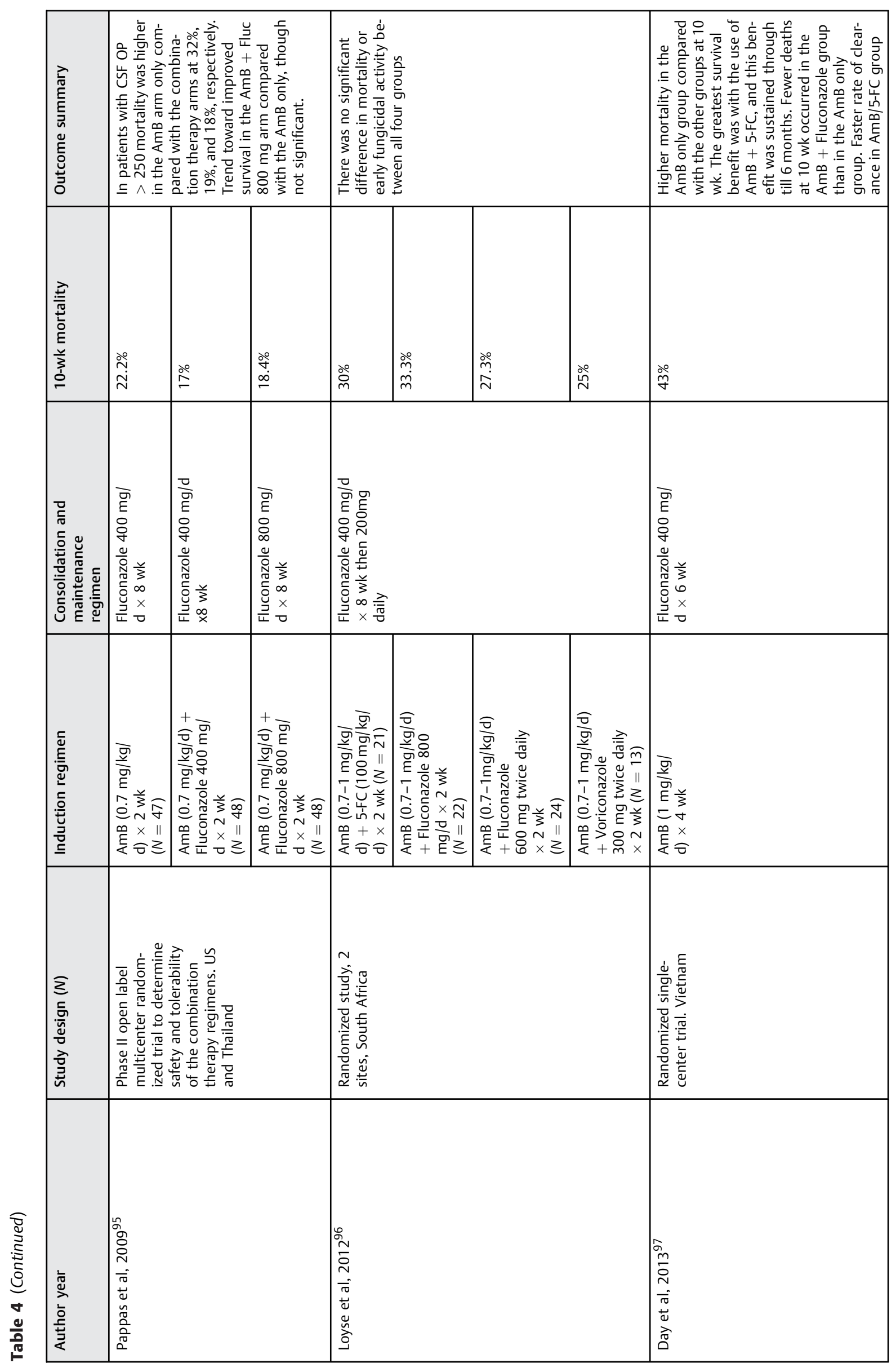




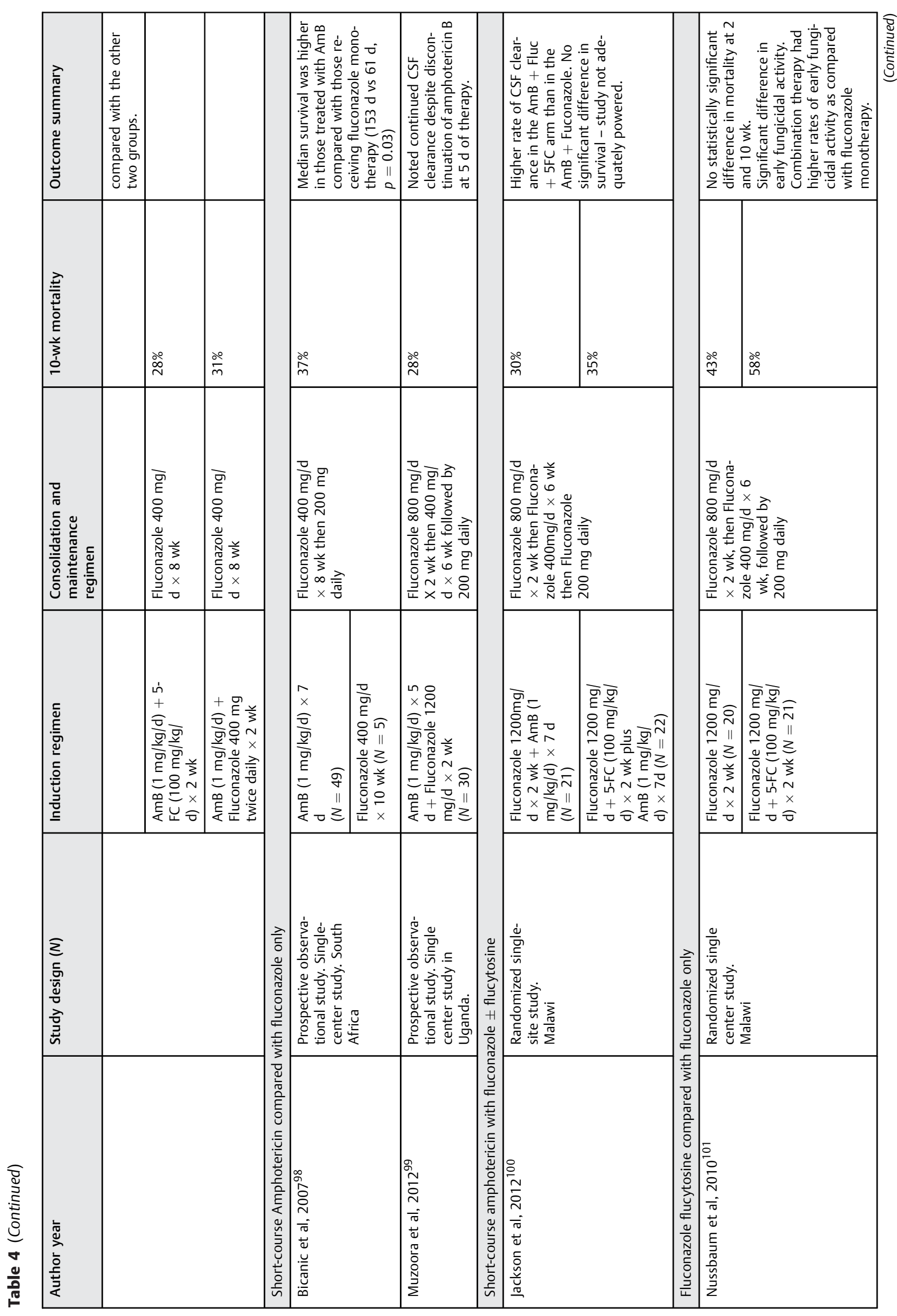




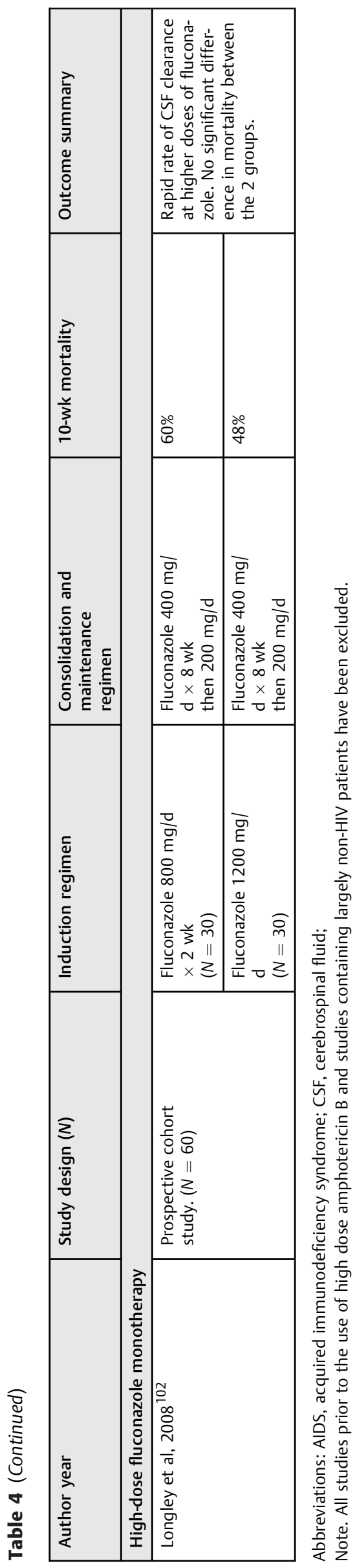

in resource-limited settings. Several studies have assessed the efficacy of alternative regimens that either contain Amphotericin B alone, amphotericin with fluconazole, fluconazole with flucytosine, fluconazole monotherapy or short courses of amphotericin B. The results of the key studies are summarized in $\mathbf{- T a b l e ~} \mathbf{4}$. The greatest limitation is that most of the studies are small, single center studies that are not adequately powered to assess mortality benefit of a given regimen. Most patients in sub-Saharan Africa continue to receive induction therapy with fluconazole monotherapy despite the associated high mortality rates. ${ }^{66}$

In addition to antifungal therapy, optimal management of raised intracranial pressure is required to reduce morbidity and mortality. Patients who have CSF opening pressures $\geq 25 \mathrm{~cm}$ of $\mathrm{H}_{2} \mathrm{O}$ should undergo repeat lumbar puncture until CSF pressures have normalized. Daily lumbar punctures may be required. If pressures remain persistently elevated a lumbar drain should be considered. $^{67}$

\section{Management of Toxicities Associated with Cryptococcal Meningitis Treatment}

Amphotericin has significant associated toxicities. These include headaches, chills, fever, and some local site reactions that can be minimized by the use of analgesics as well as slow infusion of amphotericin B over 4 to 6 hours. Renal toxicities are common and may result in significant decreases in glomerular filtration rate, necessitating early termination of the drug. Renal dysfunction will typically resolve after discontinuation of amphotericin $B .{ }^{68}$ Other renal associated toxicities include hypokalemia and hypomagnesaemia. Ample prehydration with isotonic fluid is recommended to minimize amphotericin-induced nephrotoxicity. Monitoring of electrolytes should be done at least twice weekly, with appropriate replacement of potassium and magnesium. Anemia is another common side effect of amphotericin $B$ therapy, ${ }^{69}$ and monitoring of hemoglobin at least once a week during amphotericin B therapy is recommended. Flucytosine can lead to marrow toxicity resulting in neutropenia, thrombocytopenia, anemia, or pancytopenia. Discontinuation of the drug will often result in resolution. Finally, interactions between therapies directed against cryptococcal meningitis and those targeted to treat comorbidities and other infections (i.e., tuberculosis) pose significant challenges to optimal pharmacologic therapy and must be monitored.

\section{Duration of Maintenance Therapy}

Fluconazole maintenance therapy is recommended to prevent recurrence. ${ }^{70}$ Maintenance therapy should be given with fluconazole $200 \mathrm{mg}$ orally once daily. Maintenance therapy or secondary prophylaxis should be continued until immune reconstitution and viral suppression have been achieved. ${ }^{71,72}$ Typically prophylaxis should be continued until the viral load is suppressed and the CD4 count is $>200$ cells $/ \mathrm{mm}^{3}$ for at least 3 to 6 months. 


\section{Diagnosis and Treatment of Cryptococcal Immune Reconstitution Inflammatory Syndrome}

Cryptococcal immune reconstitution inflammatory syndrome (IRIS) can occur in 8 to $49 \%$ of individuals with HIV starting ART. ${ }^{73}$ IRIS can occur as a paradoxical reaction in those with known disease who are either currently on effective therapy or who have previously successfully completed treatment. It can also occur in the setting of "unmasking" previously undiagnosed subclinical cryptococcal disease. IRIS likely occurs as a result of a dysregulated immune response to cryptococcal antigens. ${ }^{74}$ The risk factors for developing IRIS include high fungal burden, low baseline CD4 count, early introduction of antiretroviral therapy prior to sufficient CSF sterilization, and initial rapid decline in viral load. ${ }^{75,76}$ The syndrome can occur weeks to months following initiation of ART and can be associated with significant morbidity and mortality. Treatment for IRIS includes continuation of ART and antifungal therapy, and in severe cases corticosteroids may be considered. ${ }^{67}$

\section{Timing of ART in Cryptococcal Disease}

The exact timing of ART initiation in patients with cryptococcosis and HIV coinfection is yet to be determined. In patients with opportunistic infections such as Pneumocystis jiroveci, delayed initiation of ART is associated with higher rates of AIDS progression and/or death than early initiation of ART. ${ }^{77}$ Early initiation of ART in patients with tuberculosis is associated with increased risk of IRIS, but decreased mortality, particularly in those with low CD4 counts. ${ }^{78-80}$ In patients with tuberculous meningitis, early ART initiation is not associated with improved clinical outcomes. ${ }^{81}$ Early initiation of ART in patients with cryptococcal meningitis is associated with poor clinical outcomes. In a study conducted in Zimbabwe, patients were randomized to early initiation of ART (within $72 \mathrm{~h}$ of diagnosis) or delayed initiation (at 10 wk). Patients who were initiated on ART early had significantly higher 3-year mortality than those in whom ART was delayed ( $88 \%$ vs. $54 \%, p=0.006$ ). All patients were treated with fluconazole monotherapy at $800 \mathrm{mg}$ once daily. ${ }^{82}$ Early ART was also associated with increased mortality in Ugandan patients with cryptococcal meningitis treated with amphotericin B therapy who were randomized to early ART initiation within 1 to 2 weeks of starting antifungal therapy compared with those in whom ART was delayed and initiated at 5 to 6 weeks (D Bouleware, CROI 2013 ${ }^{83}$ ). Most experts would recommend delaying initiation of ART in patients with cryptococcal meningitis and HIV infection for at least 4 weeks, particularly in patients who are treated with fluconazole monotherapy.

\section{Prevention of Cryptococcal Disease}

The greatest burden of cryptococcal disease is in resource limited settings where it is associated with HIV infection in severely immunosuppressed individuals with CD4 counts
$<100$ cells $/ \mathrm{mm}^{3,84}$ Significant reductions in cryptococcal disease incidence can be achieved by decreasing HIV incidence, improving access to ART, and initiation of ART at higher CD4 counts. In individuals who access care late at CD4 T cell counts below 100 cells $/ \mathrm{mm}^{3}$, screening for cryptococcal disease by cryptococcal antigen testing, exclusion of cryptococcal meningitis in those who screen positive, and pre-emptive treatment for asymptomatic cryptococcal disease may decrease cryptococcal associated morbidity and mortality. ${ }^{85,86}$ However, implementing these strategies prospectively has yet to show a mortality benefit. ${ }^{87}$ Where available, all individuals with CD4 counts $<100$ cells $/ \mathrm{mm}^{3}$ who are being evaluated for ART initiation should be screened for cryptococcal disease and offered appropriate therapy, although evidence for optimal therapy for asymptomatic disease detected by screening is lacking. ${ }^{88}$

In conclusion, cryptococcal meningitis remains one of the leading causes of AIDS associated death, and one of the most common fungal mycoses among immunocompromised individuals. Significant progress has been made in simplifying the diagnosis of cryptococcosis and thereby enabling diagnostic testing to be available in resource limited settings with limited laboratory infrastructure. Widespread implementation of screening programs in some sub-Saharan countries will hopefully lead to substantial decreases in the number of infected people. Further research now needs to focus on identifying cheaper, less toxic, oral antifungal agents that are as efficacious as amphotericin B and flucytosine in reducing cryptococcal associated mortality. In the interim, registration of, reducing the cost of, and increasing access to amphotericin B and flucytosine must become a priority for governments in sub-Saharan Africa.

\section{References}

1 Park BJ, Wannemuehler KA, Marston BJ, Govender N, Pappas PG, Chiller TM. Estimation of the current global burden of cryptococcal meningitis among persons living with HIV/AIDS. AIDS 2009; 23(4):525-530

2 Hakim JG, Gangaidzo IT, Heyderman RS, et al. Impact of HIV infection on meningitis in Harare, Zimbabwe: a prospective study of 406 predominantly adult patients. AIDS 2000;14(10):1401-1407

3 Scarborough M, Gordon SB, Whitty CJ, et al. Corticosteroids for bacterial meningitis in adults in sub-Saharan Africa. N Engl J Med 2007;357(24):2441-2450

4 Mwaba P, Mwansa J, Chintu C, et al. Clinical presentation, natural history, and cumulative death rates of 230 adults with primary cryptococcal meningitis in Zambian AIDS patients treated under local conditions. Postgrad Med J 2001;77(914):769-773

5 French N, Gray K, Watera C, et al. Cryptococcal infection in a cohort of HIV-1-infected Ugandan adults. AIDS 2002;16(7): 1031-1038

6 Bennett JE, Kwon-Chung KJ, Howard DH. Epidemiologic differences among serotypes of Cryptococcus neoformans. Am J Epidemiol 1977;105(6):582-586

7 Grover N, Nawange SR, Naidu J, Singh SM, Sharma A. Ecological niche of Cryptococcus neoformans var. grubii and Cryptococcus gattii in decaying wood of trunk hollows of living trees in Jabalpur City of Central India. Mycopathologia 2007;164(4): 159-170 
8 Kielstein P, Hotzel H, Schmalreck A, Khaschabi D, Glawischnig W. Occurrence of Cryptococcus spp. in excreta of pigeons and pet birds. Mycoses 2000;43(1-2):7-15

9 Kwon-Chung KJ, Bennett JE. Epidemiologic differences between the two varieties of Cryptococcus neoformans. Am J Epidemiol 1984;120(1):123-130

10 Ellis DH. Cryptococcus neoformans var. gattii in Australia. J Clin Microbiol 1987;25(2):430-431

11 Ellis DH, Pfeiffer TJ. Natural habitat of Cryptococcus neoformans var. gattii. J Clin Microbiol 1990;28(7):1642-1644

12 Galanis E, Macdougall L, Kidd S, Morshed M; British Columbia Cryptococcus gattii Working Group. Epidemiology of Cryptococcus gattii, British Columbia, Canada, 1999-2007. Emerg Infect Dis 2010;16(2):251-257. Erratum in: Emerg Infect Dis 2010;16 (4):750. Kidd, Sarah [added]; Morshed, Mohammad [added]; British Columbia Cryptococcus gattii Working Group [added]. Emerg Infect Dis 2011;17(9):1784

13 Gluck JL, Myers JP, Pass LM. Cryptococcemia due to Cryptococcus albidus. South Med J 1987;80(4):511-513

14 Melo JC, Srinivasan S, Scott ML, Raff MJ. Cryptococcus albidus meningitis. J Infect 1980;2(1):79-82

15 Kamalam A, Yesudian P, Thambiah AS. Cutaneous infection by Cryptococcus laurentii. Br J Dermatol 1977;97(2):221-223

16 Lynch JP III, Schaberg DR, Kissner DG, Kauffman CA. Cryptococcus laurentii lung abscess. Am Rev Respir Dis 1981;123(1):135-138

17 Binder L, Csillag A, Toth G. Diffuse infiltration of the lungs associated with Cryptocococcus luteolus. Lancet 1956;270(6931): 1043-1045

18 Hajjeh RA, Conn LA, Stephens DS, et al; Cryptococcal Active Surveillance Group. Cryptococcosis: population-based multistate active surveillance and risk factors in human immunodeficiency virus-infected persons. J Infect Dis 1999;179(2):449-454

19 Dromer F, Mathoulin-Pélissier S, Fontanet A, Ronin O, Dupont B, Lortholary O; French Cryptococcosis Study Group. Epidemiology of HIV-associated cryptococcosis in France (1985-2001): comparison of the pre- and post-HAART eras. AIDS 2004;18(3): 555-562

20 Bratton EW, El Husseini N, Chastain CA, et al. Comparison and temporal trends of three groups with cryptococcosis: HIV-infected, solid organ transplant, and HIV-negative/non-transplant. PLoS ONE 2012;7(8):e43582

21 Pyrgos V, Seitz AE, Steiner CA, Prevots DR, Williamson PR. Epidemiology of cryptococcal meningitis in the US: 1997-2009. PLoS ONE 2013;8(2):e56269

22 McCarthy KM, Morgan J, Wannemuehler KA, et al. Populationbased surveillance for cryptococcosis in an antiretroviral-naive South African province with a high HIV seroprevalence. AIDS 2006;20(17):2199-2206

23 Meiring ST, Quan VC, Cohen C, et al; Group for Enteric, Respiratory and Meningeal disease Surveillance in South Africa (GERMS-SA). A comparison of cases of paediatric-onset and adult-onset cryptococcosis detected through population-based surveillance, 2005-2007. AIDS 2012;26(18):2307-2314

24 Ganiem AR, Parwati I, Wisaksana R, et al. The effect of HIV infection on adult meningitis in Indonesia: a prospective cohort study. AIDS 2009;23(17):2309-2316

25 Jarvis JN, Boulle A, Loyse A, et al. High ongoing burden of cryptococcal disease in Africa despite antiretroviral roll out. AIDS 2009;23(9):1182-1183

26 Bamba S, Lortholary O, Sawadogo A, Millogo A, Guiguemdé RT, Bretagne S. Decreasing incidence of cryptococcal meningitis in West Africa in the era of highly active antiretroviral therapy. AIDS 2012;26(8):1039-1041

27 Goldman DL, Khine H, Abadi J, et al. Serologic evidence for Cryptococcus neoformans infection in early childhood. Pediatrics 2001;107(5):E66

28 Abadi J, Pirofski La. Antibodies reactive with the cryptococcal capsular polysaccharide glucuronoxylomannan are present in sera from children with and without human immunodeficiency virus infection. J Infect Dis 1999;180(3):915-919

29 Davis J, Zheng WY, Glatman-Freedman A, et al. Serologic evidence for regional differences in pediatric cryptococcal infection. Pediatr Infect Dis J 2007;26(6):549-551

30 Byrnes EJ III, Li W, Lewit Y, et al. First reported case of Cryptococcus gattii in the Southeastern USA: implications for travel-associated acquisition of an emerging pathogen. PLoS ONE 2009;4(6):e5851

31 Lindberg J, Hagen F, Laursen A, Stenderup J, Boekhout T. Cryptococcus gattii risk for tourists visiting Vancouver Island, Canada. Emerg Infect Dis 2007;13(1):178-179

32 Shao X, Mednick A, Alvarez M, van Rooijen N, Casadevall A, Goldman DL. An innate immune system cell is a major determinant of species-related susceptibility differences to fungal pneumonia. J Immunol 2005;175(5):3244-3251

33 Huffnagle GB, Yates JL, Lipscomb MF. T cell-mediated immunity in the lung: a Cryptococcus neoformans pulmonary infection model using SCID and athymic nude mice. Infect Immun 1991;59(4): 1423-1433

34 Kawakami K, Qureshi MH, Zhang T, Okamura H, Kurimoto M, Saito A. IL-18 protects mice against pulmonary and disseminated infection with Cryptococcus neoformans by inducing IFN-gamma production. J Immunol 1997;159(11):5528-5534

35 Miyagi K, Kawakami K, Kinjo Y, et al. CpG oligodeoxynucleotides promote the host protective response against infection with Cryptococcus neoformans through induction of interferon-gamma production by CD4+ T cells. Clin Exp Immunol 2005;140(2):220-229

36 Jarvis JN, Casazza JP, Stone HH, et al. The phenotype of the Cryptococcus-specific CD4+ memory T-cell response is associated with disease severity and outcome in HIV-associated cryptococcal meningitis. J Infect Dis 2013;207(12):1817-1828

37 Lortholary O, Improvisi L, Rayhane N, et al. Cytokine profiles of AIDS patients are similar to those of mice with disseminated Cryptococcus neoformans infection. Infect Immun 1999;67(12): 6314-6320

38 Garcia-Hermoso D, Janbon G, Dromer F. Epidemiological evidence for dormant Cryptococcus neoformans infection. J Clin Microbiol 1999;37(10):3204-3209

39 Saha DC, Goldman DL, Shao X, et al. Serologic evidence for reactivation of cryptococcosis in solid-organ transplant recipients. Clin Vaccine Immunol 2007;14(12):1550-1554

40 Ieong MH, Reardon CC, Levitz SM, Kornfeld H. Human immunodeficiency virus type 1 infection of alveolar macrophages impairs their innate fungicidal activity. Am J Respir Crit Care Med 2000; 162(3 Pt 1):966-970

41 Chuck SL, Sande MA. Infections with Cryptococcus neoformans in the acquired immunodeficiency syndrome. N Engl J Med 1989; 321(12):794-799

42 Moosa MY, Coovadia YM. Cryptococcal meningitis in Durban, South Africa: a comparison of clinical features, laboratory findings, and outcome for human immunodeficiency virus (HIV)-positive and HIV-negative patients. Clin Infect Dis 1997;24(2):131-134

43 Heyderman RS, Gangaidzo IT, Hakim JG, et al. Cryptococcal meningitis in human immunodeficiency virus-infected patients in Harare, Zimbabwe. Clin Infect Dis 1998;26(2):284-289

44 Lee SC, Dickson DW, Casadevall A. Pathology of cryptococcal meningoencephalitis: analysis of 27 patients with pathogenetic implications. Hum Pathol 1996;27(8):839-847

45 Graybill JR, Sobel J, Saag M, et al; The NIAID Mycoses Study Group and AIDS Cooperative Treatment Groups. Diagnosis and management of increased intracranial pressure in patients with AIDS and cryptococcal meningitis. Clin Infect Dis 2000;30(1):47-54

46 Johnston SR, Corbett EL, Foster O, Ash S, Cohen J. Raised intracranial pressure and visual complications in AIDS patients with cryptococcal meningitis. J Infect 1992;24(2):185-189

47 Crump JR, Elner SG, Elner VM, Kauffman CA. Cryptococcal endophthalmitis: case report and review. Clin Infect Dis 1992; 14(5):1069-1073 
48 Saha DC, Xess I, Jain N. Evaluation of conventional \& serological methods for rapid diagnosis of cryptococcosis. Indian J Med Res 2008;127(5):483-488

49 Thiruchelvan N, Wuu KY, Arseculeratne SN, Ashraful-Haq J. A pseudo-cryptococcal artefact derived from leucocytes in wet India ink mounts of centrifuged cerebrospinal fluid. J Clin Pathol 1998;51(3):246-248

50 Lee SC, Casadevall A, Dickson DW. Immunohistochemical localization of capsular polysaccharide antigen in the central nervous system cells in cryptococcal meningoencephalitis. Am J Pathol 1996;148(4):1267-1274

51 Butler WT, Alling DW, Spickard A, Utz JP. Diagnostic and prognostic value of clinical and laboratory findings in cryptococcal meningitis, a follow-up study of forty patients. N Engl J Med 1964; 270:59-67

52 Bloomfield N, Gordon MA, Elmendorf DF Jr. Detection of Cryptococcus neoformans antigen in body fluids by latex particle agglutination. Proc Soc Exp Biol Med 1963;114(1):64-67

53 Bennett JE, Hasenclever HF, Tynes BS. Detection of cryptococcal polysaccharide in serum and spinal fluid: value in diagnosis and prognosis. Trans Assoc Am Physicians 1964;77:145-150

54 Tanner DC, Weinstein MP, Fedorciw B, Joho KL, Thorpe JJ, Reller L. Comparison of commercial kits for detection of cryptococcal antigen. J Clin Microbiol 1994;32(7):1680-1684

55 Hamilton JR, Noble A, Denning DW, Stevens DA. Performance of cryptococcus antigen latex agglutination kits on serum and cerebrospinal fluid specimens of AIDS patients before and after pronase treatment. J Clin Microbiol 1991;29(2):333-339

56 Gade W, Hinnefeld SW, Babcock LS, et al. Comparison of the PREMIER cryptococcal antigen enzyme immunoassay and the latex agglutination assay for detection of cryptococcal antigens. J Clin Microbiol 1991;29(8):1616-1619

57 Binnicker MJ, Jespersen DJ, Bestrom JE, Rollins LO. Comparison of four assays for the detection of cryptococcal antigen. Clin Vaccine Immunol 2012;19(12):1988-1990

58 Lindsley MD, Mekha N, Baggett HC, et al. Evaluation of a newly developed lateral flow immunoassay for the diagnosis of cryptococcosis. Clin Infect Dis 2011;53(4):321-325

59 Jarvis JN, Percival A, Bauman S, et al. Evaluation of a novel pointof-care cryptococcal antigen test on serum, plasma, and urine from patients with HIV-associated cryptococcal meningitis. Clin Infect Dis 2011;53(10):1019-1023

60 Gates-Hollingsworth MA, Kozel TR. Serotype sensitivity of a lateral flow immunoassay for cryptococcal antigen. Clin Vaccine Immunol 2013;20(4):634-635

61 Hansen J, Slechta ES, Gates-Hollingsworth MA, et al. Large-scale evaluation of the immuno-mycologics lateral flow and enzymelinked immunoassays for detection of cryptococcal antigen in serum and cerebrospinal fluid. Clin Vaccine Immunol 2013;20(1): 52-55

62 Lawn SD, Wood R. Point-of-care urine antigen screening tests for tuberculosis and cryptococcosis: potential for mortality reduction in antiretroviral treatment programs in Africa. Clin Infect Dis 2012;54(5):739-740

63 Bennett JE, Dismukes WE, Duma RJ, et al. A comparison of amphotericin $B$ alone and combined with flucytosine in the treatment of cryptoccal meningitis. N Engl J Med 1979;301(3): 126-131

64 Hospenthal DR, Bennett JE. Flucytosine monotherapy for cryptococcosis. Clin Infect Dis 1998;27(2):260-264

65 Spickard A, Butler WT, Andriole V, Utz JP. The improved prognosis of cryptococcal meningitis with amphotericin B therapy. Ann Intern Med 1963;58:66-83

66 Rothe C, Sloan DJ, Goodson P, et al. A prospective longitudinal study of the clinical outcomes from cryptococcal meningitis following treatment induction with 800 . $\mathrm{mg}$ oral fluconazole in Blantyre, Malawi. PLoS ONE 2013;8(6):e67311
67 Perfect JR, Dismukes WE, Dromer F, et al. Clinical practice guidelines for the management of cryptococcal disease: 2010 update by the Infectious Diseases Society of America. Clin Infect Dis 2010; 50(3):291-322

68 Bicanic T, Wood R, Meintjes G, et al. High-dose amphotericin B with flucytosine for the treatment of cryptococcal meningitis in HIV-infected patients: a randomized trial. Clin Infect Dis 2008; 47(1):123-130

69 Miller RP, Bates JH. Amphotericin B toxicity. A follow-up report of 53 patients. Ann Intern Med 1969;71(6):1089-1095

70 Bozzette SA, Larsen RA, Chiu J, et al; California Collaborative Treatment Group. A placebo-controlled trial of maintenance therapy with fluconazole after treatment of cryptococcal meningitis in the acquired immunodeficiency syndrome. N Engl J Med 1991;324(9):580-584

71 Kirk O, Reiss P, Uberti-Foppa C, et al; European HIV Cohorts. Safe interruption of maintenance therapy against previous infection with four common HIV-associated opportunistic pathogens during potent antiretroviral therapy. Ann Intern Med 2002;137(4): 239-250

72 Vibhagool A, Sungkanuparph S, Mootsikapun P, et al. Discontinuation of secondary prophylaxis for cryptococcal meningitis in human immunodeficiency virus-infected patients treated with highly active antiretroviral therapy: a prospective, multicenter, randomized study. Clin Infect Dis 2003;36(10):1329-1331

73 Haddow LJ, Colebunders R, Meintjes G, et al; International Network for the Study of HIV-associated IRIS (INSHI). Cryptococcal immune reconstitution inflammatory syndrome in HIV-1infected individuals: proposed clinical case definitions. Lancet Infect Dis 2010;10(11):791-802

74 Boulware DR, Meya DB, Bergemann TL, et al. Clinical features and serum biomarkers in HIV immune reconstitution inflammatory syndrome after cryptococcal meningitis: a prospective cohort study. PLoS Med 2010;7(12):e1000384

75 Shelburne SA, Visnegarwala F, Darcourt J, et al. Incidence and risk factors for immune reconstitution inflammatory syndrome during highly active antiretroviral therapy. AIDS 2005;19(4):399-406

76 Skiest DJ, Hester LJ, Hardy RD. Cryptococcal immune reconstitution inflammatory syndrome: report of four cases in three patients and review of the literature. J Infect 2005;51(5):e289-e297

77 Zolopa A, Andersen J, Powderly W, et al. Early antiretroviral therapy reduces AIDS progression/death in individuals with acute opportunistic infections: a multicenter randomized strategy trial. PLoS ONE 2009;4(5):e5575

78 Havlir DV, Kendall MA, Ive P, et al; AIDS Clinical Trials Group Study A5221. Timing of antiretroviral therapy for HIV-1 infection and tuberculosis. N Engl J Med 2011;365(16):1482-1491

79 Blanc FX, Sok T, Laureillard D, et al; CAMELIA (ANRS 1295-CIPRA KH001) Study Team. Earlier versus later start of antiretroviral therapy in HIV-infected adults with tuberculosis. N Engl J Med 2011;365(16):1471-1481

80 Abdool Karim SS, Naidoo K, Grobler A, et al. Integration of antiretroviral therapy with tuberculosis treatment. $\mathrm{N}$ Engl J Med 2011;365(16):1492-1501

81 Török ME, Yen NT, Chau TT, et al. Timing of initiation of antiretroviral therapy in human immunodeficiency virus (HIV)-associated tuberculous meningitis. Clin Infect Dis 2011;52(11): 1374-1383

82 Makadzange AT, Ndhlovu CE, Takarinda K, et al. Early versus delayed initiation of antiretroviral therapy for concurrent HIV infection and cryptococcal meningitis in sub-Saharan Africa. Clin Infect Dis 2010;50(11):1532-1538

83 Boulware D et al. ART initiation within the first 2 weeks of cryptococcal meningitis is associated with higher mortality: a multisite randomized trial. 20th Conference on Retroviruses and Opportunistic Infections; March 3-6, 2013; Atlanta, GA. Oral abstract 144 
84 Pongsai P, Atamasirikul K, Sungkanuparph S. The role of serum cryptococcal antigen screening for the early diagnosis of cryptococcosis in HIV-infected patients with different ranges of CD4 cell counts. J Infect 2010;60(6):474-477

85 Meya DB, Manabe YC, Castelnuovo B, et al. Cost-effectiveness of serum cryptococcal antigen screening to prevent deaths among HIV-infected persons with a CD4 + cell count $<$ or $=100$ cells/ microL who start HIV therapy in resource-limited settings. Clin Infect Dis 2010;51(4):448-455

86 Jarvis JN, Lawn SD, Vogt M, Bangani N, Wood R, Harrison TS. Screening for cryptococcal antigenemia in patients accessing an antiretroviral treatment program in South Africa. Clin Infect Dis 2009;48(7):856-862

87 Meyer AC, Kendi CK, Penner JA, et al. The impact of routine cryptococcal antigen screening on survival among HIV-infected individuals with advanced immunosuppression in Kenya. Trop Med Int Health 2013;18(4):495-503

88 Meyer AC, Jacobson M. Asymptomatic cryptococcemia in resource-limited settings. Curr HIV/AIDS Rep 2013;10(3): 254-263

89 World Health Organization. Rapid advice: diagnosis, prevention and management of cryptococcal disease in HIV-infected adults, adolescents and children. http://www.who.int/hiv/pub/cryptococcal_disease2011/.2011. Accessed November 20, 2013

90 Govender NP, Meintjes G, Bicanic T, et al. Guideline for the prevention, diagnosis and management of cryptococcal meningitis among HIV infected persons: 2013 update. S Afr J HIV Med 2013;14(2):76-86

91 Guidelines for the prevention and treatment of opportunistic infections in HIV-infected adults and adolescents: recommendations from the Centers for Disease Control and Prevention, the National Institutes of Health, and the HIV Medicine Association of the Infectious Diseases Society of America. http://aidsinfo.nih. gov/contentfiles/adult_oi.pdf. 2013. Accessed November 20, 2013

92 van der Horst CM, Saag MS, Cloud GA, et al; National Institute of Allergy and Infectious Diseases Mycoses Study Group and AIDS Clinical Trials Group. Treatment of cryptococcal meningitis associated with the acquired immunodeficiency syndrome. N Engl J Med 1997;337(1):15-21
93 Hamill RJ, Sobel JD, El-Sadr W, et al. Comparison of 2 doses of liposomal amphotericin B and conventional amphotericin B deoxycholate for treatment of AIDS-associated acute cryptococcal meningitis: a randomized, double-blind clinical trial of efficacy and safety. Clin Infect Dis 2010;51(2):225-232

94 Brouwer AE, Rajanuwong A, Chierakul W, et al. Combination antifungal therapies for HIV-associated cryptococcal meningitis: a randomised trial. Lancet 2004;363(9423):1764-1767

95 Pappas PG, Chetchotisakd P, Larsen RA, et al. A phase II randomized trial of amphotericin $B$ alone or combined with fluconazole in the treatment of HIV-associated cryptococcal meningitis. Clin Infect Dis 2009;48(12):1775-1783

96 Loyse A, Wilson D, Meintjes G, et al. Comparison of the early fungicidal activity of high-dose fluconazole, voriconazole, and flucytosine as second-line drugs given in combination with amphotericin B for the treatment of HIV-associated cryptococcal meningitis. Clin Infect Dis 2012;54(1):121-128

97 Day JN, Chau TT, Wolbers M, et al. Combination antifungal therapy for cryptococcal meningitis. N Engl J Med 2013; 368(14):1291-1302

98 Bicanic T, Meintjes G, Wood R, et al. Fungal burden, early fungicidal activity, and outcome in cryptococcal meningitis in antiretroviral-naive or antiretroviral-experienced patients treated with amphotericin B or fluconazole. Clin Infect Dis 2007;45(1):76-80

99 Muzoora CK, Kabanda T, Ortu G, et al. Short course amphotericin B with high dose fluconazole for HIV-associated cryptococcal meningitis. J Infect 2012;64(1):76-81

100 Jackson AT, Nussbaum JC, Phulusa J, et al. A phase II randomized controlled trial adding oral flucytosine to high-dose fluconazole, with short-course amphotericin B, for cryptococcal meningitis. AIDS 2012;26(11):1363-1370

101 Nussbaum JC, Jackson A, Namarika D, et al. Combination flucytosine and high-dose fluconazole compared with fluconazole monotherapy for the treatment of cryptococcal meningitis: a randomized trial in Malawi. Clin Infect Dis 2010;50(3):338-344

102 Longley N, Muzoora C, Taseera K, et al. Dose response effect of high-dose fluconazole for HIV-associated cryptococcal meningitis in southwestern Uganda. Clin Infect Dis 2008;47(12):1556-1561 